The atomic nucleus provides an unique and fascinating object for studying complexity owing to its extended compact structure, the strength of quantum effects and the delicate balance between a Coulomb force of infinite range and a short range nuclear force.

\title{
Atomic Nuclei: Laboratories for Studying Complexity
}

Fundamental research on complexity will impact upon areas as diverse as biology, weather prediction, and the regulation of stock markets. One is now beginning to discover the fascinating richness of self-organization in apparently disordered systems and the immense wealth of moderately broken symmetries emerging from large collections of individually simple systems.

Studying the nucleus is mandatory if we are to understand the nature of matter lying between elementary particles and atoms and molecules. It is legitimate to consider whether nuclei can be understood from the standpoint of complexity. Conversely, we can ask the question: does the atomic nucleus provide a laboratory for probing the behavior of complex systems? We shall argue that nuclear physics does indeed offer excellent opportunities... without getting ourselves lost.

\section{Nuclei as Sets of Interacting Nucleons}

We shall focus on conventional nuclear systems, where only protons and neutrons, and a few photons and mesons are present. This choice is not completely arbitrary. The most microscopic constituents of nuclei are presumably the quarks and virtual gluons which nucleons and mesons are made of. It could be argued that these fundamental particles correspond better to what one would call the elementary constituents of the system. But it turns out that quarks and gluons are for the most part tightly bound in intermediate structures, mainly in the nucleons. In this sense nucleons are the effective elementary bricks which constitute "normal" nuclei. In order to detect a signature of the presence of quarks one has to excite nuclear matter so much that nuclei as such do not exist anymore. These exceptional thermodynamic conditions may have existed during the early life of the universe, but present-day nuclei may safely be viewed as collections of interacting nucleons.

Nuclei interpolate between systems with a few degrees of freedom ( $N=3$ for instance) and systems of Avogadro size $\left(N \approx 10^{23}\right)$.

Y. Abe joined the research staff of Hokkaido University, Japan, on graduating in 1964. He has been a professor at the Yukawa Institute for Theoretical Physics since 1975.

B.G. Giraud graduated from the Ecole Polytechnique, Paris, in 1961 and has worked at the Commissariat à l'Energie Atomique, Saclay, since 1965.

M. Ploszajczak studied in Cracow and Bonn and joined the Institute of Nuclear Physics, Cracow, in 1981 before moving to GANIL, Caen, in 1990.

E. Suraud has been a professor at the University of Toulouse since 1992. He received his Ph.D. from the University of Paris in 1984 and then worked at Grenoble University and GANIL.

\section{Y. Abe', B.G. Giraud ${ }^{2}$, M. Ploszajczak ${ }^{3}$, E. Suraud ${ }^{1,4}$}

${ }^{1}$ Yukawa Institute for Theoretical Physics, Kyoto University, Japan

${ }^{2}$ Service de Physique Théorique, CE-Saclay

${ }^{3}$ Grand Accélérateur National d'lons Lourds (GANIL), Caen

${ }^{4}$ Laboratoire de Physique Quantique, Université Paul Sabatier, Toulouse

As a matter of fact, the richness of phenomena shown by nuclei when $N$ runs from a few units to a few hundreds is staggering: 20 to 30 years ago, the beauty of the shell model [1] and the collective model [2] as efficient simplifications of this many-body problem exemplified what basic science can do to make the real world intelligible. More recently, it has been found that order parameters describing the shell structure are still useful when studying very excited states such as superdeformed nuclei [3] and new kinds of giant resonances [4].

\section{Complex Nuclear Systems}

In drawing attention to the complexity of nuclear dynamics we shall first briefly look back to the early days of nuclear physics. We shall see that a stochastic picture of nucleonic motion inside the nucleus is almost as old as nuclear physics itself. In spite of the symmetries of the mean field in which nucleons move, the stochastic picture basically remains valid and reflects the complex nature of nuclear dynamics. Indeed, experiments performed during the 1980's at new heavy-ion facilities all over the world have shown that this complex picture is the rule rather than an exception.

We shall also present a contemporary example of a heavy-ion reaction exhibiting typical stochastic features. The questions raised demonstrate the liveliness of this field of research (Fig. 1), while the techniques used to address the problems are of general interest outside nuclear physics.

From an experimental point of view, physicists have learned to build detectors able to keep track of thousands of projectiles. Moreover, they have gained considerable experience in analyzing large bodies of data and extracting from them relevant physical information. On the other hand, the theoretical description of complex heavy-ion reactions requires the development and simulation of microscopic, dynamical models in strongly out-of-equilibrium situations. We shall try to describe some benchmarks for present and future tasks.

\section{Early Stochastic Models}

Niels Bohr writing in Nature in 1936 is probably responsible for introducing statistical concepts into the description of a nuclear problem, namely the interaction of a neutron with a target nucleus. A statistical picture of the numerous interactions of the incident neutron with the nucleons constituting the nucleus basically reflects the complexity of the process. In the case of moderately energetic neutrons, such as those available in the mid-1930's, the entire interaction pro-

Fig. 1-The INDRA detector at GANIL features a high geometrical efficiency ( $\approx 90 \%$ coverage of the $4 \pi$ solid angle) and low values of the energy threshold $(\approx 1 \mathrm{MeV}$ per nucleon). The multidetector system comprises 336 detector cells of various sizes to give an approximately uniform counting rate. Their arrangement provides a mass identification of light charged particles and charge identification of fragments (up to $Z=30$ ). Experiments designed to investigate among other topics, the onset of multifragmentation as a function of the size of the nuclear system, started in March 1993 using A, Xe and Gd beams bombarding various targets.

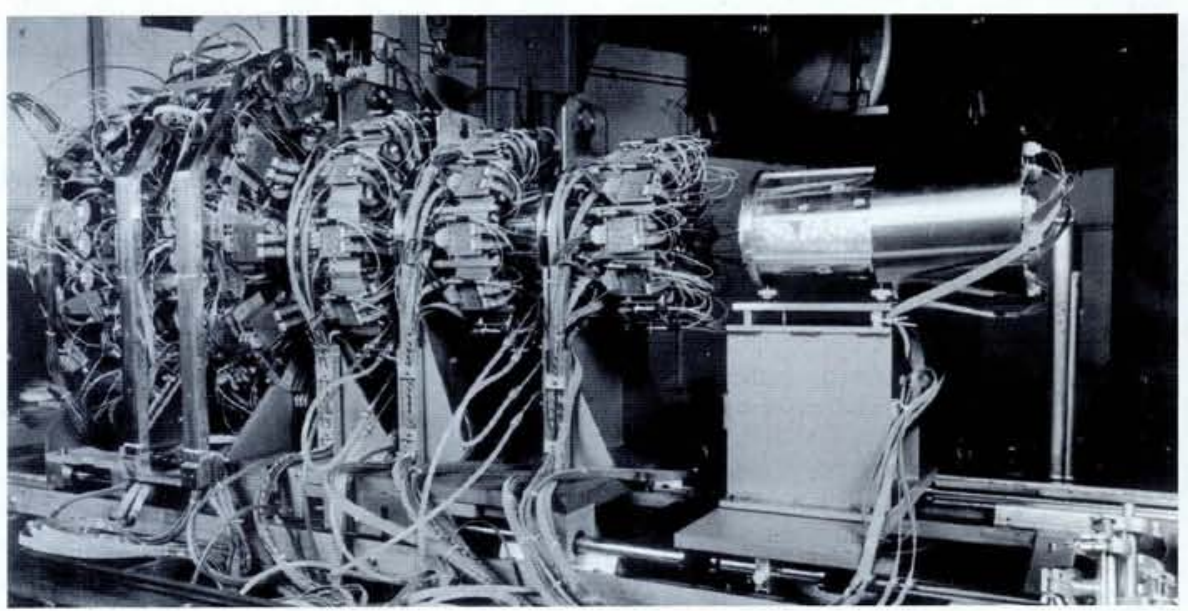




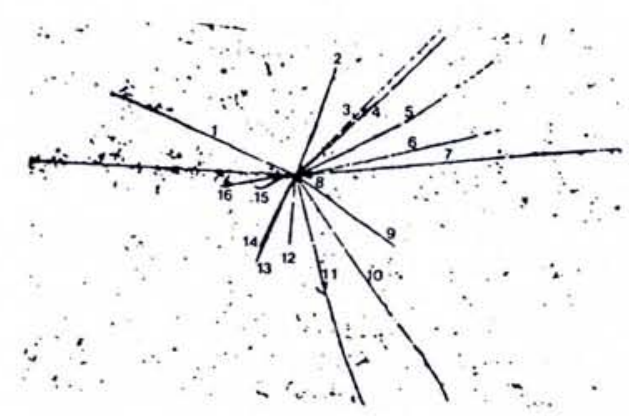

cess is very long relative to nuclear times, and leads to the formation of an excited nucleus whose subsequent decay may be reliably described using a statistical approach.

At the end of the decade, Kramers in a landmark paper [5] on the Langevin description of chemical reactions suggested that the newly discovered fission phenomenon might also be viewed as a diffusion process of a collective variable (e.g., the deformation of the nucleus) over a potential barrier. This description of a nuclear process once again implied the underlying assumption of complicated nucleonic motion, which as a first step was treated as a heat bath with respect to the deformation considered as a Brownian particle. So complexity lay at the heart of the overall phenomenological description of the physical situation.

\section{The Beauty of the Shell Model}

But apparent regularity was also a feature of nucleonic motion. Indeed, the introduction of the shell model in the late 1940's by Mayer and Jensen shed a new light on the motion of nucleons inside a nucleus [1]. To a good approximation, nucleonic motion could be represented as nucleons following single-particle trajectories related to the levels of an unique, common, one-body potential. The shells (degeneracies with energy gaps between them) can be understood as corresponding to classical closed orbits in the potential, and are thereby related to its symmetry properties [6]. Incidentally, this concept is also fully applicable to another finite fermion system, namely microclusters, where it is further developed to account for "supershells" owing to interference between two nearby orbits. All told, the shell model suggested an underlying regularity rather than complexity - a picture that was apparently difficult to reconcile with the earlier stochastic one.

The paradox was in fact short-lived since Wigner soon realized [7] that the structure of nuclear spectra was much richer than that given by a simple textbook exercise in quantum mechanics. By studying the spacings of compound nucleus levels he showed that the distribution of spacings was special, representing a signature of a restricted class of stochastic Hamiltonians. As a side product, his remarks gave birth to today's most widely recognized definition of quantum chaos.

Spectra of compound nucleus levels provide the best experimental data for statistical studies of quantum chaos [8]. So once again nucleonic motion is associated with stochasticity, at least at high excitation energies, thus reflecting the complex nature of nuclei.
Fig. 2 - The collision of a carbon ion of $852 \mathrm{MeV}$ in a nuclear emulsion. Some 16 fragments with charges $Z$ between 1 and 4 have been identified. From [9].

\section{Modern Nuclear Complexity: \\ The Physics of Violent Collisions}

The beauty of the nuclear shell model enlightened nuclear physics in the 1960's and 1970's and allowed the development of original contributions to the physics of strongly interacting many-body systems. The Hartree-Fock mean-field theory permitted the description of many ground-state nuclear properties, with the help of realistic phenomenological effective interactions. The dynamical version of this theory, the socalled time-dependent Hartree-Fock (TDHF) theory, however soon suffered from deficiencies in the description of nuclear collisions as bombarding energies increased in magnitude.

The mean-field picture using the approach Bohr had developed was basically unable to account for the dissipation responsible for the heating of nuclei, which experimentalists were starting to observe in fusion-like reactions of heavy ions at beam energies of a few tens of $\mathrm{MeV}$ per nucleon. Once again, statistical concepts were taken up, this time in terms of thermodynamic preoccupations such as the determination of the equation of state for nuclear matter. This obliged nuclear physicists to depart from a mean-field picture and to accommodate the observed dissipative patterns. The existence of so-called nuclear temperatures also implied the occurrence of corresponding thermal fluctuations, thus bringing the nuclear physics community back to basic questions concerning the statistical mechanics of complex systems. Moreover, it soon turned out that many of the properties of atomic nuclei, such as the nuclear equation of state and transport properties, could in fact only be studied in strongly out-of-equilibrium processes. These processes, in turn, exhibited unique features that called for further study.

\section{The Dynamics of Multifragmentation}

The dynamics of nuclear fragmentation in heavy-ion collisions is an example of a situation where a discussion of both chaotic structures and complexity owing to multiparticle correlations may be especially relevant. In heavy-ion collisions at beam velocities of the order of the average velocity of nucleons in a nucleus at rest, the system created may break up into several nuclei of intermediate mass (see Fig. 2). Physicists have sought for many years for a signal of a liquid-gas phase transition of a finite system in such collisions. Whatever the underlying mechanism, its time-scale is of the same order as the typical time-scales of the system. The process has thus to be analyzed in terms of dynamics, and the road to a proper theoretical description of multifragmentation was, and remains, tortuous.

The first step is to depart from the conforting mean-field picture by accommodating dissipative features. This is a very challenging task at the quantum level so a generally accepted solution to the problem has not yet been found. Fortunately, the de Broglie wavelengths of nucleons inside colliding heavy ions very soon become negligibly small on increasing the bombarding energy. This allows one to rely on semiclassical descriptions which are relatively simple as compared to those formulated in a quantum framework.

During the 1980's, a nuclear version of Boltzmann's equation was used with success in the context of the semi-classical model [10]. It turned out that although a complete theoretical justification of such an equation might still be missing, its application to actual heavy-ion collisions helped greatly in understanding the basic dynamical process. In particular, it allowed one to account for, with a reasonable degree of confidence, the formation of "hot" nuclei (compound systems characterised by temperatures exceeding a few $\mathrm{MeV}$ resulting from incoherent excitations). The dynamics of multifragment formation, however, could not be fully explained within such a theoretical framework, which by its very nature only predicts average quantities.

The next step in the development of these microscopic theories was to extend basic kinetic equations into a stochastic regime [13]. Stochastic extensions of kinetic equations, introduced in the late 1950's, had been studied in fact during the 1960's and '70's in situations close to equilibrium. They were now developed and applied in strongly out-of-equilibrium contexts, and in situations where self-consistent mean-field effects still play an important rôle. Such analyses are presently the subject of many investigations, both experimental and theoretical. Combined with elaborate statistical descriptions of the decay properties of the fragments produced during collisions, they may eventually provide a microscopic theory of multifragment emission.

The relevance of this research extends beyond understanding fragment formation. This is because these dynamic systems offer interesting clues to the general question of the correspondence between the quantum and classical regimes. As implied above, variations of the beam energy in a certain sense amount to tuning an effective Planck constant. On the other hand, the self-consistent nature of the nuclear mean field makes the basic equations of motion non-linear, which brings us back to chaotic features. This non-linearity seems to be unavoidable as it stems from a reduction of the many-body problem to one-body dynamics.

\section{From Statistical to Non-statistical \\ Patterns}

It is well known that the flow of a classical incompressible fluid, which for low viscosities is laminar, exhibits highly chaotic and irregular behaviour at high viscosities. In this viscous regime, flow is both unstable and unpredictable: a small perturbation at a given time may rapidly lead to a strong dis- 
tortion of the flow pattern. An understanding of a large number of statistical properties of the fluid can be obtained by fragmenting at different scales the turbulent structures, called "eddies", into smaller and smaller eddies. This random cascade of fragmenting eddies provides the principle physical mechanism by which the energy of the flow is transmitted to motion at smaller scales down to almost the molecular scale, where dissipation halts further fragmentation.

In heavy-ion collisions, one expects that hot, expanding nuclear matter may enter a region where density perturbations of finite length become unstable [12]. Inside these regions of mechanical instability, called spinodal instabilities, the system is thermodynamically unstable with respect to fluctuations having small wavelengths and it is doubtful that Boltzmann-like kinetic equations, which neglect high-order correlations, continue to be valid approximations. The picture emerging from these considerations resembles that of a critical phenomenon involving a random cascade of fragmentations with unstable blobs of nuclear matter at different scales. For some specific scaling fragmentation functions of this non-equilibrium cascade process, the distribution of fragment sizes may reach a limit reminiscent of those distributions produced by percolation [13]. This leads at once to the following questions: is the nuclear fragmentation process related to a critical phenomenon? And if so, what is the nature of this

\section{Percolation}

A percolation model comprises a static array of points (sites) which can be occupied randomly with a probability for any site being independent of the probability for neighbours. Certain occupied sites are said to be linked if they are at adjacent points on the lattice (site percolation) or if the bonds between adjacent points are active (site-bond percolation) In the latter case, the clusters are given by a sequence of occupied sites connected by active bonds.

Consider the situation with all lattice sites occupied and the links between them randomly activated (i.e., bond percolation). When the bond activation probability is close to zero, most sites will be isolated or form small clusters. On the other hand, when the probability is large, almost all sites are linked and a single percolating (infinite) cluster exists. The critical point in the infinite system arises when 0.25 of all bonds are active and one finds large fluctuations in both the size of clusters as well as in their multiplicity.

The transition from a non-percolating phase to a percolating one is a general model for a second-order phase transition. The essential ingredients of a percolation calculation are the randomness of the linkages, the locality of the breaking process involved in the cluster formation, and the short-range nature of the forces. The same ingredients are assumed to be the basic elements of a percolation model for nuclear fragmentation. finite-size phase transition, and under which kinematic conditions of the collision is the dynamics of the fragmenting matter governed by the criticality?

At the present time, experiments with an exclusive analysis, event by event, of the fragment-size distribution are restricted to those employing nuclear emulsion techniques and only a limited number of fully resolved events are at our disposal. However, questions related to non-equilibrium cascading will soon be studied in great detail using electronic techniques based on sophisticated multidetector systems now operational at GANIL (Caen, France: Fig. 1), GSI (Darmstadt, Germany) and Michigan State University (East Lansing, USA).

Existing data strongly suggest that a critical phenomenon is indeed involved. The way to identify it is by analyzing observable quantities which behave in a qualitatively different manner when a critical phenomenon is present. The next step is to compare these analyses with models, such as nonequilibrium random cascading or percolation (see insert), where the nature of the "finite size phase transition" is well understood.

Critical phenomena are usually associated with symmetry breaking and large fluctuations. In the nuclear case, since the distribution of the cluster distribution is not directly measurable it has been proposed to look for a signal of critical behaviour in the cluster size distribution [14]. In Fig. 3 we show an example of such an analysis for the dimensionless quantity $\gamma_{2}=M_{2} M_{0} / M_{1}^{2}$ plotted versus the reduced multiplicity $m_{0}$ for:

(a, upper) experimental data on the fragmentation of gold nuclei (with incident energies of approximately $1 \mathrm{GeV} /$ nucleon) following interactions in a nuclear emulsion:

(b, lower) three-dimensional random-bond percolation on a cubic lattice, which in an infinite lattice exhibits a second-order phase transition when $m_{0} \rightarrow 0.25$.

The fragment-size distribution is calculated in each event by the factorial moments $M_{k}=\Sigma_{z} Z^{k} n_{Z}$, where $k>0$ and the sum runs over all fragment charges (sizes) excluding the largest one. The value $\gamma_{2} \gg 2$ indicates a power law distribution of the fragment sizes, as expected around a critical point. The distributions plotted in Fig. 3 show that large values of $\gamma_{2}$ are found around $m_{0} \approx$ 0.25 in both the experimental data and the three-dimensional percolation analysis.

\section{Concluding Remarks}

The theories of percolation and non-equilibrium random cascading for fragmentation, as well as the exclusive analysis of the multifragment events, have helped in developing the idea that randomness and disorder associated with heavy-ion collisions may result in characteristic features that are remarkable $[14,15]$. These features are not merely the result of statistical fluctuations of otherwise continuous, smooth spectra of fragments, but represent a genuine physical effect related to the scaling features of the nuclear dynamics.

One is naturally led to study distributions, such as those given by the theory of fractals, which satisfy the observed anomalous scaling laws and reflect the scale invariance of an underlying geometry or chaotic dyna- mics. This leads to new types of kinetic theories and a new version of the statistical mechanics of small systems in strongly outof-equilibrium situations, with the possibility of dynamic instabilities and spontaneous pattern formation.

Let us risk a speculation: nothing prevents a priori a local density fluctuation in a soup of nucleons from propagating and even selfreplicating within the nucleaus. The challenge is to understand the dynamic evolution of these fluctuations at the microscopic level. Further development of stochastic extensions of kinetic equations may offer a method to attack this fundamental problem.

There is probably a long way to go before all these problems can be correctly formulated and solved. But even at the present stage of this development it may be helpful to discuss the atomic nucleus as an intrinsically dynamic object. Its extended compact structure, the strength of quantum effects and the delicate balance of a Coulomb force of infinite range and a short-range nuclear force make the atomic nucleus an unique and fascinating object for research on complexity. Theoretical and experimental work is now helping to explain nuclear collisions in regimes where classical limits and residual quantum mechanisms compete and collective and isolated degrees of freedom
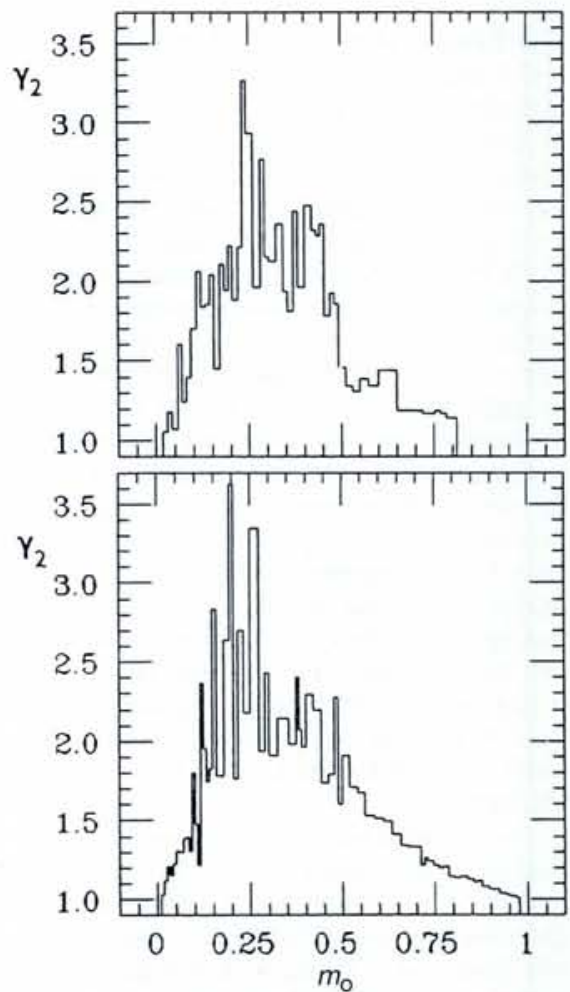

Fig. 3 - The dimensionless quantity $\gamma_{2}$ for: (a, upper) experimental data describing to the fragmentation of gold nuclei with the incident energy of $1 \mathrm{GeV} /$ nucleon in a nuclear emulsion; $(b$, lower) three-dimensional random bond percolation on a cubic lattice. The data consist of about 400 events for which charges of all fragments were measured. The percolation calculation was carried by setting the number of occupied sites equal to the number of nucleons in the fragmenting gold nucleus. The bond activation parameter $q$ for each separate percolation event was chosen at random $(0<q<1)$. From [14]. 


\section{More Light on Random Number Generators}

coexist, with many cases of interference, intermittency, stability, and instability.

A conceptual framework which could transfer the experience gained in nuclear physics to other fields is still missing. Interdisciplinary experts are not so numerous, and there remains much to be done until solutions to many-body problems in nuclear physics could be useful in biology, sociology or economics. Nonetheless, it can be safely claimed that atomic nuclei, and more specifically nuclear collisions, provide a frame of reference for the study of complexity that is unparalleled in the richness of its phenomenology. It would be a serious mistake to overlook this opportunity.

[1] Haxel O., Jensen J.H.D. and Suess H.E., Phys. Rev. 75 (1949) 1766; Mayer M.G., Phys. Rev. 74 (1948) 235; ibid. 75 (1949) 1969.

[2] Bohr N. and Kalckar K., Mat. Fys. Medd. Dan. Vid. Selsk. 14 (1937) No. 10; Bohr A. Mat. Fys. Medd. Dan Vid. Selsk. 26 (1952) No. 14; A. Bohr A. and Mottelson B.R., Mat. Fys. Medd. Dan. Vid. Selsk. 27 (1953) No. 16.

[3] Twin P.J. et al., Phys. Rev. Lett. 57 (1986) 811.

[4] Hansen P.G. and Johnson B., Europhys. Lett. 4 (1987) 409.

[5] Kramers H.A., Physica VII 4 (1940) 284.

[6] Balian R. and Bloch C., Ann. Phys. (New York) 69 (1972) 76.

[7] Wigner E.P., Ann. Math. 62 (1955) 548.

[8] Bohigas O. and Weidenmüller H.A., Ann.

Rev. Nucl. Part. Sci. 38 (1988) 421.

[9] Jakobsson B. et al, Z. Physik A 307 (1982) 293.

[10] Bertsch G. and Das Gupta S., Phys. Rep. 160 (1988) 189.

[11] Ayik S. and Grégoire Ch., Phys. Lett. B 212 (1988) 269.

[12] Heiselberg H., Pethick C.J. and Ravenhall D.G., Phys. Rev. Lett. 61 (1988) 818.

[13] Botet R. and Ploszajczak M., Phys. Rev. Lett. 69 (1992) 3696.

[14] Campi X., J. Phys. A 19 (1986) L917; Phys. Lett. B 208 (1988) 351.

[15] Ploszajczak M. and Tucholski A., Phys. Rev. Lett. 65 (1990) 1539.
Considerable activity has been triggered by recent results of Monte Carlo calculations showing that random number generators considered good were giving the wrong answers in Ising model simulations using the highly efficient Wolff algorithm [see EN 24 (1993) 24].

Until recently, the only pseudorandom number generators which had been studied extensively were those of the multiplicative congruential type; they present a well-known defect whose effect can be made arbitrarily small at the expense of some computing time, and there is no wider theory which excludes their having additional as yet unknown defects. For this reason, many Monte Carlo practitioners prefer to use random numbers generated using other algorithms which produce much longer sequences before repeating and do not have the known defect of congruential generators. Unfortunately, Ferrenberg et al. [Phys. Rev. Lett. 69 (1992) 3382] showed that for certain problems these newer generators gave wrong answers. Now Martin Lüscher of DESY has studied one of these generators, the "subtract-with-borrow" algorithm of Marsaglia and Zaman known as RCARRY, and was able to show how to improve its chaotic properties.

As Lüscher says: "The characteristic feature of a chaotic dynamical system is that trajectories starting at nearby states diverge exponentially with time. Even if the evolution is locally continuous, such a system appears to behave randomly on larger time scales. One could also say that any state specified to some finite precision has an exponentially deteriorating memory of its history." Although RCARRY is "strongly chaotic" in this sense, it has short-term correlations which can be eliminated by a simple modification.

The RCARRY algorithm works on a table of 24 floating-point numbers and produces its next number by combining two of the numbers in the table and replacing one of them by the new result. Lüscher therefore

\section{Postdoctoral Fellowships \\ at the}

\section{Niels Bohr Institute}

The Niels Bohr Institute is part of the physics department of Copenhagen University and has active research programmes in theoretical and experimental particle physics, nuclear physics, physics of nonlinear and complex systems, cluster physics and theoretical and observational astrophysics. The Institute is host for two independent research institutes: Nordita (Nordic Institute for Theoretical Physics) and NBA (Niels Bohr Archive) and three research centres: TAC (Center for Theoretical Astrophysics), CATS (Center for Chaos and Turbulence Studies) and Connect (Center for Neural Network Studies), and there is a close collaboration with the scientists of these institutions. The Niels Bohr Institute has active programmes on research facilities such as the CERN accelerators, ESO and NOT telescopes, and the NORDBALL detector.

All applications for positions at the Niels Bohr Institute will be considered under the Institute's own international programmes. Applicants who are citizens of EC member states (or countries treated as member states) are eligible for fellowships at the "Niels Bohr Institute EC Physics Training Center". This is supported by the European Community "Human Capital and Mobility" programme.

Applicants should include a curriculum vitae, list of publications and a statement of their research interests and goals. In addition, applicants should arrange for 2-3 letters of reference to be sent directly. All material should be sent to:

Postdoctoral Positions Office, Ulla Holm, Secretary

The Niels Bohr Institute, Blegdamsvej 17, DK-2100 Copenhagen Ø, Denmark.

Tel: (+45) 35325272 - Fax: (+45) 31421016 - Email: uhclm@nbi.dk

The deadline for applications for the academic year 1994/95 is 12 December 1993 .

\section{RANLUX}

A subroutine implementing the skipping algorithm called RANLUX (for luxury random numbers) has been written by $F$. James and is being introduced into the CERN Program Library. The program, along with the paper of M. Lüscher [DESY preprint 93-133] will be published in Computer Physics Communications. Subroutine RANLUX allows the user to choose the "luxury level" between zero (no skipping, same as RCARRY) and four (skipping 365). Level two already passes all known tests and gives the right answers for the Wolff algorithm, but users who want an even better guarantee of security and can afford the extra computer time (about a factor 2.5 at level two and 5 at level four) can opt for pure luxury. For many problems, where the random number generation time is small compared with the rest of the calculation, there is no longer any reason for the computational physicist to deny himself the luxury of random numbers with demonstrably good chaotic properties.

looked at it as a generator of vectors in the 24-dimensional hypercube, and considered how two neighboring vectors in this hypercube would evolve according to the algorithm. He showed that indeed nearby trajectories would diverge exponentially, on the average with a Lyapunov exponent close to one, that is the separation would increase by about a factor $e$ every cycle of 24 numbers. After about 16 cycles, the smallest possible separation $\left(6 \times 10^{-8}\right)$ would grow to be of order one and fill all the hypercube.

In order to accelerate this "chaotic explosion", he suggested a modification of the algorithm whereby one would generate 24 numbers and then skip a certain number (at most 365 ) before using 24 more. This "throwing away" of random numbers is normally to be avoided since it shortens the effective period of a generator, but Lüscher also showed that if the total number generated and skipped per cycle is prime, the period is maintained. (In fact, since the basic period of RCARRY is about $10^{170}$, one can afford to shorten the period by many orders of magnitude without any danger of exhausting it.)

F. James, CERN, Geneva

\section{ERRATA}

Some mistakes crept into last month's anniversary issue of Europhysics News. The Editor apologises for the following:

- The group shown on the cover is the Conference Committee and not the Publications Committee.

- E. Gotsman from Tel Aviv University shown in the photo on p. 120 is clearly not a "Turkish politician".

- The Condensed Matter general conference had a "new beginning" in 1986 and not 1980 (p. 125).

- J.M. Charap was the Chairman and not the Secretary of High Energy Physics in 1981-84 (p. 126).

- Seated to the right of W. Buckel in the photo on p. 150 is L.A. Radicati and not G. Szigeti. 